Research Article

\title{
Robust Fixed-Time Synchronization for Coupled Delayed Neural Networks with Discontinuous Activations Subject to a Quadratic Polynomial Growth
}

\author{
Lina Yu $\mathbb{D}^{\mathbb{D}},{ }^{1}$ Yunfei Ma $\mathbb{D}^{1},{ }^{1}$ Yuntong Yang $\mathbb{D}^{1},{ }^{1,2}$ Jingchao Zhang $\mathbb{D},{ }^{1}$ and Chunwei Wang $\mathbb{D}^{1,3}$ \\ ${ }^{1}$ School of Information Science and Engineering, Yanshan University, Qinhuangdao 066004, China \\ ${ }^{2}$ School of Electronics Science, Northeast Petroleum University, Daqing 163318, Heilongjiang, China \\ ${ }^{3}$ Beijing Branch, Daqing Oilfield Information Technology Company, Beijing 100043, China
}

Correspondence should be addressed to Chunwei Wang; wangchunwei@stumail.ysu.edu.cn

Received 26 November 2020; Revised 22 January 2021; Accepted 2 February 2021; Published 17 February 2021

Academic Editor: Sanbo Ding

Copyright (C 2021 Lina Yu et al. This is an open access article distributed under the Creative Commons Attribution License, which permits unrestricted use, distribution, and reproduction in any medium, provided the original work is properly cited.

In this paper, we focus on the robust fixed-time synchronization for discontinuous neural networks (NNs) with delays and hybrid couplings under uncertain disturbances, where the growth of discontinuous activation functions is governed by a quadratic polynomial. New state-feedback controllers, which include integral terms and discontinuous factors, are designed. By Lyapunov-Krasovskii functional method and inequality analysis technique, some sufficient criteria, which ensue that networks can realize the robust fixed-time synchronization, are addressed in terms of linear matrix inequalities (LMIs). Moreover, the upper bound of the settling time, which is independent on the initial values, can be determined to any desired values in advance by the configuration of parameters in the proposed control law. Finally, two examples are provided to illustrate the validity of the theoretical results.

\section{Introduction}

In recently years, coupled neural networks (CNNs), as a special sort of complex dynamic networks, have attracted the widespread attention from a lot of scholars due to its potential applications in parallel computation, multiagent cooperative control, cryptography, nuclear magnetic resonance instrument, and other aspects [1-5]. Particularly, the synchronization with respect to CNNs has been extensively studied in many science fields [6-11] and the references therein.

Time delay often arises in the transmission of signals in CNNs [12, 13]. In [13], Shao and Zhang considered the delay-dependent stabilization for CNNs with two additive input delays. In [14], He et al. investigated the pinning synchronization for CNNs with hybrid couplings and delays by an adoptive approach. In [15], Wang and Huang discussed the pining synchronization of delayed CNNs with reaction-diffusion effects.
Recently, many works are devoted to the synchronization behaviors of NNs with discontinuous activations. For example, in [16], by applying the state-feedback control strategies, the global finite-time synchronization conditions are addressed for delayed NNs with discontinuous activations. In [17], the authors discussed the global synchronization for NNs with time-varying delays and discontinuous right-hand side. The exponential synchronization for discontinuous NNs with delays has been considered in [18]. In [19], the discrete nonfragile control strategy was designed to achieve the synchronization for fractional-order NNs by adjusting the coupling gain.

In engineering applications, the synchronization is required to be achieved in a finite time [20, 21]. In [22], the authors pointed out that the finite-time control can demonstrate better disturbance and robustness rejection properties. Therefore, it is meaningful to investigate the global synchronization in finite time for CNNs [23-27]. 
However, it should be noted that the upper bound of the settling time greatly depends on the initial states of $\mathrm{CNNs}$ under the finite-time synchronization. In generality, it is difficult to obtain the initial values in the real world. This shows that there exists a constraint to practical applications of CNNs [28]. To solve this problem, the fixed-time synchronization control strategy is considered in [28], where the settling time is independent on the initial conditions of CNNs. At present, as a kind of efficient control strategies, the fixed-time synchronization control has received tremendous attention from many researchers. The global fixed-time synchronization was discussed for semi-Markovian jumping neural networks with time-varying delays and discontinuous activation functions in [29]. The authors discussed the global fixed-time stability of dynamical nonlinear systems and realized the global fixed-time synchronization for CNNs with discontinuous activations in [30]. In [31], the nonsingular fixed-time consensus tracking was considered for second-order multiagent networks. Then, Zheng et al. discussed the fixed-time synchronization of memristive fuzzy BAM cellular neural networks with time-varying delays based on feedback controllers in [32]. Some criterions were obtained for the fixed-time synchronization of CohenGrossberg CNNs with and without parameter uncertainties in [33]. By fixed-time controllers, the global synchronization of delayed hybrid CNNs is studied in [34]. In [35, 36], Zhu et al. discussed the synchronization in fixed time with hybrid couplings and delays for semiMarkovian switching complex NNs, and the upper bound of settling time could be determined under the designed controller. The authors considered the global synchronization in fixed time for CNNs with delays and discontinuous/continuous activations and proposed two discontinuous control protocols in [37]. Event-triggered synchronization in fixed time was investigated for semiMarkov switching dynamical complex networks with multiple weights and discontinuous nonlinearity in [38]. It is worth pointing out, to the best of authors' knowledge, there are few results on the global synchronization for hybrid CNNs with discontinuous activations subject to a nonlinear function growth.

Inspired by the above discussion, in this paper, we focus on the global robust fixed-time synchronization for hybrid CNNs with delays and discontinuous activations in the presence of disturbances. Under the designed controllers with integral terms and discontinuous factors, the global synchronization conditions in fixed time are addressed in the terms of LMIs. Moreover, the upper bound of the settling time is estimated explicitly. The primary contributions of this paper are listed as follows:

(1) The neuron activation functions are modeled to be discontinuous and subject to a quadratic polynomial growth.

(2) On the basis of the configuration of parameters in the designed control law, the upper bound of the settling time can be determined to any desired values in advance.

(3) The robust synchronization conditions in fixed time are achieved in the form of LMIs.

The rest of this paper is organized as follows. In Section 2, some preliminaries and CNNs model are provided. In Section 3, the state-feedback discontinuous controllers are designed, and the global fixed-time synchronization conditions are addressed in the form of LMIs. In Section 4, two numerical simulations verifying the theoretic findings are presented. Conclusion is received in Section 5.

Notation. $R$ refers to the set of real numbers. $R^{n}$ represents the $n$-dimensional Euclidean space, and $R^{n \times n}$ denotes the set of all $n \times n$ real matrices. Given $A \in R^{n \times n}, \lambda_{\max (A)}$ $\left(\lambda_{\min (A)}\right)$ stands for the maximal (minimal) eigenvalues of $A$. $A>0(A<0)$ represents $A$ is a positive (negative) definite matrix. Set $\eta=\left(\eta_{1}, \eta_{2}, \ldots, \eta_{n}\right)^{T} \in R^{n}$, where the superscript $T$ is the transpose operator. Define $\|\eta\|_{q}=\left(\sum_{i=1}^{n}\left|\eta_{i}\right|^{q}\right)^{(1 / q)}$, $|\eta|^{\alpha}=\left(\left|\eta_{1}\right|^{\alpha},\left|\eta_{2}\right|^{\alpha}, \ldots,\left|\eta_{n}\right|^{\alpha}\right)^{T}, \quad$ and $\operatorname{sig}^{\alpha}(\eta)=\left(\left|\eta_{1}\right|^{\alpha}\right.$ $\left.\operatorname{sign}\left(\eta_{1}\right)\right),\left(\left|\eta_{2}\right|^{\alpha} \operatorname{sign}\left(\eta_{2}\right), \ldots,\left|\eta_{n}\right|^{\alpha} \operatorname{sign}\left(\eta_{n}\right)\right)^{T}$, where $\alpha$ and $q$ are positive constants and $|\cdot|$ denotes the absolute real value. For a set $\Phi \subset \mathbb{R}^{n}, \overline{c o}[\Phi]$ represents the closure of the convex hull of $\Phi$. Let $A, B, C$, and $D$ be matrices with appropriate dimensions. $\otimes$ denotes the Kronecker product.

\section{Preliminaries and System Description}

Consider an array of hybrid CNNs described by

$$
\begin{aligned}
\dot{x}_{i}(t)= & -Q x_{i}(t)+A f\left(x_{i}(t)\right)+B f\left(x_{i}(t-\tau)\right)+c_{1} \sum_{j=1}^{N} d_{i j} x_{j}(t) \\
& +c_{2} \sum_{j=1}^{N} h_{i j} x_{j}(t-\tau)+J(t)+u_{i}(t)+\Delta_{i}\left(t, x_{i}(t)\right),
\end{aligned}
$$

where $i=1,2, \ldots, N, x_{i}(t)=\left(x_{i 1}(t), x_{i 2}(t), \ldots, x_{i n}(t)\right)^{T}$ is the state of the $i$ th network at time $t$; $x(t)=\left(x_{1}^{T}(t), x_{2}^{T}(t), \ldots, x_{N}^{T}(t)\right)^{T}, \quad \mathrm{Q}=\operatorname{diag}\left(q_{1}, \ldots, q_{n}\right)$, $q_{i}>0 ; A=\left(a_{l r}\right)_{n \times n}$ represents the connection weight matrix; $B=\left(b_{l r}\right)_{n \times n}$ denotes the delayed connection matrix; $c_{1}$ and $c_{2}$ are couple strength; $f\left(x_{i}(t)\right)=\left(f_{1}\left(x_{i 1}(t)\right)\right.$, $\left.\ldots, f_{n}\left(x_{i n}(t)\right)\right)^{T}$ intends neuron activation function; $\tau>0$ is delay. $u_{i}(t)$ stands for the control input; $J(t)=\left(J_{1}(t), J_{2}(t), \ldots, J_{n}(t)\right)^{T}$ is an external input; and $D=\left(d_{i j}\right)_{N \times N}$ and $H=\left(h_{i j}\right)_{N \times N}$ indicate coupling configuration matrix and delayed coupling configuration matrix, respectively. If there exists an edge from node $i$ to $j$, then $d_{i j}=d_{j i}>0$; otherwise, $d_{i j}=d_{j i}=0(i \neq j)$. Laplacian matrix $L=\left(l_{i j}\right)_{N \times N}$ of a graph corresponding to $D$ is given by $l_{i i}=\sum_{j=1, j \neq i}^{N} d_{i j}, l_{i j}=-d_{i j}, i \neq j . \Delta_{i}$ expresses the uncertain disturbance.

In system $(1), f_{i}(\cdot)$ is made to satisfy the following assumptions:

(A1) $f_{i}(\cdot)$ is continuous expect on a countable set of isolate points $\rho_{k}^{i}$, and $f_{i}(\cdot)$ has at most a limited number of discontinuous points on any compact 
interval of $R$; in addition, at the discontinuous points $\rho_{k}^{i}$, the finite right limit $f_{i}\left(\rho_{k}^{i+}\right)$ and left limit $f_{i}\left(\rho_{k}^{i-}\right)$ exist.

(A2) Let $\widehat{D} \in R^{n}$ be a domain containing the origin. There exist positive real constants $\nu_{l}, \omega_{l}$, and $g_{l}$ for each $l=1,2, \ldots, n, i=1,2, \ldots, N, \forall t \geq 0$, such that

$$
\begin{aligned}
& \sup \left|\gamma_{i l}(t)-\widetilde{\gamma}_{l}(t)\right| \leq g_{l}\left|x_{i l}(t)-y_{l}(t)\right|^{2} \\
& \quad+v_{l}\left|x_{i l}(t)-y_{l}(t)\right|+\omega_{l},
\end{aligned}
$$

holds for $\forall x_{i l}(t), y_{l}(t) \in \widehat{D}$, where $\tilde{\gamma}_{l}(t) \in \overline{c o}\left[f_{l}\left(y_{l}(t)\right)\right], \gamma_{i l} \in \overline{c o}\left[f_{l}\left(x_{i l}(t)\right)\right]$.

Based on $\mathrm{A} 1$, it follows that $\overline{c o}\left[f\left(x_{i}\right)\right]=\left(\overline{c o}\left[f_{1}\left(x_{i 1}\right)\right]\right.$, $\left.\left.\overline{c o}\left[f_{2}\left(x_{i 2}\right)\right], \ldots, \overline{c o}\left[f_{n}\left(x_{i n}\right)\right]\right)^{T}, \quad \overline{c o}\left[f_{n}\left(x_{i n}\right)\right]\right)^{T}$, where $\overline{c o}\left[f_{l}\left(x_{i l}\right)\right]=\left[\min \left\{f_{i}\left(x_{i l}^{-}\right), f_{i}\left(x_{i l}^{+}\right)\right\}, \max \left\{f_{i}\left(x_{i l}^{-}\right), f_{i}\left(x_{i l}^{+}\right)\right\}\right]$.

Under A1, system (1) is a functional differential equation with discontinuous right-hand side [38]. In this paper, analogous to $[39,40]$, we use the definition of Filippov solutions for system (1).

Definition 1 (see [41]). $x_{i}(t)$ is solution of system (1) in Filippov sense, if the following holds:

(i) $x_{i}(t)$ is continuous on $[-\tau, T)$, and $x_{i}(t)$ is absolutely continuous on $[0, T)$.

(ii) for a.e. $t \in[0, T)$.

$$
\begin{aligned}
\dot{x}_{i}(t) \in F\left(x_{i}\right)= & -Q x_{i}(t)+A \overline{c o}\left[f\left(x_{i}(t)\right)\right] \\
& +B \overline{c o}\left[f\left(x_{i}(t-\tau)\right)\right]+c_{1} \sum_{j=1}^{N} d_{i j} x_{j}(t) \\
& +c_{2} \sum_{j=1}^{N} h_{i j} x_{j}(t-\tau)+J(t) \\
& +\overline{c o}\left[u_{i}(t)\right]+\Delta_{i}\left(t, x_{i}(t)\right) .
\end{aligned}
$$

Noting that set-valued map $F\left(x_{i}\right)$ has a nonempty, compact, and convex value and is upper semicontinuous, so it is measurable. By measurable selection theorem [41], there exists measurable function $\gamma_{i}(t)=\left(\gamma_{i 1}(t), \ldots, \gamma_{\text {in }}(t)\right)^{T}$ : $[-\tau, T) \longrightarrow R^{n}, \quad \in \in \overline{c o}\left[f\left(x_{i}(t)\right)\right] \quad$ and $\quad \xi_{i}(t)=\left(\xi_{i 1}(t)\right.$, $\left.\xi_{i 2}(t), \ldots, \xi_{\text {in }}(t)\right)^{T}:[-\tau, T) \longrightarrow R^{n}, \in \in \overline{c O}\left[u_{i}(t)\right]$ such that, for a.e., $t \in[0, T)$,

$$
\begin{aligned}
\dot{x}_{i}(t)= & -Q x_{i}(t)+A \gamma_{i}(t)+B \gamma_{i}(t-\tau)+J(t)+\xi_{i}(t) \\
& +c_{1} \sum_{j=1}^{N} d_{i j} x_{j}(t) \\
& +c_{2} \sum_{j=1}^{N} h_{i j} x_{j}(t-\tau)+\Delta_{i}\left(t, x_{i}(t)\right) .
\end{aligned}
$$

Definition 2 (IVP [42]). For any $\varphi_{i}=\left(\varphi_{i 1}, \varphi_{i 2}, \ldots, \varphi_{i N}\right)^{T}$ : $[-\tau, 0] \longrightarrow R^{N}$ and any measurable selection $\phi_{i}=\left(\phi_{i 1}, \phi_{i 2}\right.$, $\left.\ldots, \phi_{i N}\right)^{T}:[-\tau, 0] \longrightarrow R^{n}$, where $\phi_{i}(s) \in \overline{c o}\left[f_{i}\left(\phi_{i}(s)\right)\right]$ for a.e $s \in[-\tau, 0]$. Absolute continuous function $x_{i}(t)=x_{i}$ $(t, \varphi, \phi)$ associated with measurable function $\gamma_{i}(t) \in \overline{c o}\left[f\left(x_{i}(t)\right)\right]$ is said to be solution of IVP of system (1) on $[0, T)$ with initial value $\left(\varphi_{i}(s), \phi_{i}(s)\right)$ if

$$
\left\{\begin{array}{l}
\dot{x}_{i}(t)=-Q x_{i}(t)+A \gamma_{i}(t)+B \gamma_{i}(t-\tau)+c_{1} \sum_{j=1}^{N} d_{i j} x_{j}(t) \\
+c_{2} \sum_{j=1}^{N} h_{i j} x_{j}(t-\tau)+J(t)+\xi_{i}(t)+\Delta_{i}\left(t, x_{i}(t)\right), \\
\quad \text { for a.e. } t \in[0, T) \\
x_{i}(s)=\widetilde{\varphi}_{i}(s), \quad \forall s \in[-\tau, 0], \\
\gamma_{i}(s)=\widetilde{\phi}_{i}(s), \quad \text { for a.e. } s \in[-\tau, 0] .
\end{array}\right.
$$

Consider the following isolated neural network:

$$
\dot{y}(t)=-Q y(t)+A f(y(t))+B f(y(t-\tau))+J(t) .
$$

Analogous to Definition 2, the IVP associated with system (6) is obtained as follows:

Definition 3 (IVP [42]). For any $\psi=\left(\psi_{1}, \psi_{2}, \ldots\right.$, $\left.\psi_{N}\right)^{T}:[-\tau, 0] \longrightarrow R^{n}$ and any measurable selection $\omega=\left(\omega_{1}, \omega_{2}, \ldots, \omega_{N}\right)^{T}:[-\tau, 0] \longrightarrow R^{n}$, where $\omega_{i}(s) \in \overline{c o}$ $\left[f_{i}\left(\psi_{i}(s)\right)\right]$ for a.e. $s \in[-\tau, 0]$. Absolute continuous function $y(t)=y(t, \psi, \omega)$ associated with measurable function $\widetilde{\gamma}(t) \in \overline{c o}[f(y(t))]$ is said to be solution of IVP of system (6) on $[0, T)$ with initial value $(\psi(s), \omega(s))$ if

$$
\left\{\begin{array}{l}
\dot{y}(t)=-Q y(t)+A \widetilde{\gamma}(t)+B \widetilde{\gamma}(t-\tau)+J(t), \\
\quad \text { for a.e. } t \in[0, T), \\
y(s)=\widetilde{\psi}(s), \quad \forall s \in[-\tau, 0], \\
\widetilde{\gamma}(s)=\omega(s), \quad \text { for a.e. } s \in[-\tau, 0] .
\end{array}\right.
$$

In this paper, our objective is to design new feedback controllers to realize the robust fixed-time synchronization between CNNs (1) and isolated network (6).

Set synchronization error $e_{i}(t)=x_{i}(t)-y(t)$. Then,

$$
\begin{aligned}
\dot{e}_{i}(t)= & -Q e_{i}(t)+A \tilde{f}\left(e_{i}(t)\right)+B \tilde{f}\left(e_{i}(t-\tau)\right)+c_{1} \sum_{j=1}^{N} d_{i j} e_{j}(t) \\
& +c_{2} \sum_{j=1}^{N} h_{i j} e_{j}(t-\tau)+u_{i}(t)+\Delta_{i}\left(t, x_{i}(t)\right),
\end{aligned}
$$

where $\tilde{f}_{i}(t)=f\left(x_{i}(t)\right)-f(y(t))$ and $\tilde{f}_{i}(t-\tau)=f\left(x_{i}(t-\right.$ $\tau))-f(y(t-\tau))$

According to Definitions 2 and 3, IVP of error system can be written as 


$$
\left\{\begin{array}{l}
\dot{e}_{i}(t)=-Q e_{i}(t)+A \widehat{\gamma}_{i}(t)+B \widehat{\gamma}_{i}(t-\tau)+\xi_{i}(t)+c_{1} \sum_{j=1}^{N} d_{i j} e_{j}(t) \\
+c_{2} \sum_{j=1}^{N} h_{i j} e_{j}(t-\tau)+\Delta_{i}\left(t, x_{i}(t)\right) \\
\quad \text { for a.e. } t \in[0, T), \\
e_{i}(s)=\widetilde{e}_{i}(s), \quad \forall s \in[-\tau, 0] \\
\hat{\gamma}_{i}(s)=\tilde{\eta}_{i}(s), \quad \text { for a.e. } s \in[-\tau, 0]
\end{array}\right.
$$

where $\widehat{\gamma}_{i}(t)=\gamma_{i}(t)-\tilde{\gamma}(t) \in \overline{c o}\left[f\left(x_{i}(t)\right)\right]-\overline{c o}[f(y(t))]$ $\in \widetilde{f}\left(e_{i}(t)\right), \widetilde{e}_{i}(s)=\widetilde{\varphi}_{i}(s)-\widetilde{\psi}(s)$, and $\widetilde{\eta}_{i}(s)=\widetilde{\phi}_{i}(s)-\omega(s)$.

In order to derive the robust synchronization results of CNNs (1), for the terms $\Delta_{i}(t, x)$, we make the following assumption:

(A3) The uncertain disturbances $\Delta_{i}(t, x)$ are bounded by

$$
\left|\Delta_{i}(t, x)\right| \leq \Delta_{\max }
$$

where $\Delta_{\max }$ is a known nonnegative constant.

Definition 4. Under the designed controller $u_{i}(t)$, if there exists time function $T\left(\tilde{e}_{i}, \tilde{\eta}_{i}\right)>0$ such that $\lim _{t \longrightarrow T\left(\tilde{e}_{i}, \tilde{\eta}_{i}\right)}\left\|e_{i}(t)\right\|=0$ and $\left\|e_{i}(t)\right\| \equiv 0, t \geq T\left(\tilde{e}_{i}, \tilde{\eta}_{i}\right)$, then system (1) is said to be globally robust finite-time synchronized with system (6). Moreover, if there exists scalar $T_{\max }>0$ such that $T\left(\tilde{e}_{i}, \tilde{\eta}_{i}\right) \leq T_{\max }$, then system (1) is said to be globally robust fixed-time synchronized with system (6). $T\left(\widetilde{e}_{i}, \tilde{\eta}_{i}\right)$ is called as the settling time function, and $T_{\max }$ is the upper bound of the settling time function.

Definition 5 (see [43]). For function $\mathscr{V}\left(x_{i}\right): R^{n} \longrightarrow R$, if

(i) $\mathscr{V}\left(x_{i}\right)$ is regular in $R^{n}$,

(ii) for $x_{i}=0, \mathscr{V}(0)=0$, and for $x_{i} \neq 0, \mathscr{V}\left(x_{i}\right)>0$,

(iii) $\mathscr{V}\left(x_{i}\right) \longrightarrow+\infty$ as $\left\|x_{i}\right\| \longrightarrow+\infty$,

then $\mathscr{V}\left(x_{i}\right)$ is called as $C$-regular.

Lemma 1 (chain rule [44]). If function $\mathscr{V}\left(x_{i}\right)$ is C-regular and $x_{i}(t)$ is absolutely continuous on $[0,+\infty)$, then $\mathscr{V}\left(x_{i}(t)\right)$ is differentiable for $x_{i}(t) t \in[0,+\infty)$ and

$$
\frac{\mathrm{d}}{\mathrm{d} t} \mathscr{V}\left(x_{i}(t)\right)=v^{T} x_{i}(t), \quad \forall v \in \partial \mathscr{V}\left(x_{i}\right)
$$

where $\partial \mathscr{V}\left(x_{i}\right)$ is the Clarke generalized gradient of $\mathscr{V}\left(x_{i}\right)$ at $x$.

Lemma 2 (see [44]). Suppose that $\mathscr{V}: R^{n} \longrightarrow R$ is C-regular and $x_{i}(t):[0,+\infty) \longrightarrow R^{n}$ is absolutely continuous. Let $\widehat{v}_{i}(t)=\mathscr{V}\left(x_{i}(t)\right)$. If there exist a continuous function $\varsigma:[0,+\infty) \longrightarrow R$ with $\varsigma(\rangle)>0,\rangle \in(0,+\infty)$, such that

$$
\begin{aligned}
\dot{\widehat{v}}_{i}(t) & \leq-\varsigma\left(\widehat{v}_{i}(t)\right), \quad \text { for a.e. }, t>0, \\
\int_{0}^{\widehat{v_{i}}(0)} \frac{1}{\varsigma(\varrho)} \mathrm{d} \varrho & =t_{1}<+\infty,
\end{aligned}
$$

then $\lim _{t \longrightarrow t_{1}} x_{i}(t)=0$ and $x_{i}(t)=0, t \geq t_{1}$. Especially, if for all $\varrho \in(0,+\infty)$ and $\varsigma(\varrho)=M \varrho^{\mu}$, where $0<\mu<1$ and $M>0$, then $t_{1}$ can be calculated by

$$
t_{1}=\frac{\widehat{v}^{1-\mu}(0)}{M(1-\mu)}
$$

Lemma 3 (see [30]). Suppose that $\mathscr{V}: R^{n} \longrightarrow R$ is a $C$-regular function and $e_{i}(t):[0,+\infty) \longrightarrow R^{n}$ is solution with initial value $\left(\tilde{e}_{i}, \tilde{\eta}_{i}\right)$ of error system (9). If there exist constants $\widetilde{a}>0, \widetilde{b}>0, \widetilde{\delta}>0, \widetilde{k}>0$, and $\widetilde{\delta} \widetilde{k}>1$, such that

$\frac{d}{d t} \mathscr{V}\left(e_{i}(t)\right) \leq-\left(\tilde{a} \mathscr{V}^{\tilde{\delta}}\left(e_{i}(t)\right)+\tilde{b}\right)^{\tilde{k}}, \quad e_{i}(t) \in R^{n} \backslash\{0\}$, for a.e. $t>0$,

then the upper bound settling time $T\left(\tilde{e}_{i}, \tilde{\eta}_{i}\right)$ is estimated by

$$
T\left(\tilde{e_{i}}, \tilde{\eta}_{i}\right) \leq T_{\max } \triangleq \frac{1}{\widetilde{b}^{k}}\left(\frac{\tilde{a}}{\tilde{b}}\right)^{(1 / \tilde{\delta})}\left(1+\frac{1}{\widetilde{\delta} \tilde{k}-1}\right) .
$$

Lemma 4 (see $[45,46])$. Let $\delta_{1}, \delta_{2}, \ldots, \delta_{n} \geq 0, q>1$, and $p \in(0,1]$. And then,

$$
\begin{aligned}
& \sum_{i=1}^{n} \delta_{i}^{q} \geq n^{1-q}\left(\sum_{i=1}^{n} \delta_{i}\right)^{q}, \\
& \sum_{i=1}^{n} \delta_{i}^{p} \geq\left(\sum_{i=1}^{n} \delta_{i}\right)^{p} .
\end{aligned}
$$

Lemma 5 (see $[47,48])$. Let $\zeta_{1}, \zeta_{2}, \ldots, \zeta_{n} \geq 0,0<p<q$. Then,

$$
\left(\sum_{i=1}^{n} \zeta_{i}^{p}\right)^{(1 / p)} \geq\left(\sum_{i=1}^{n} \zeta_{i}^{q}\right)^{(1 / q)}
$$

\section{Main Results}

Set $\widehat{a}=\max _{l, r \in[1, n]}\left|a_{l r}\right|, \widehat{b}=\max _{\tilde{L}_{l, r \in[1, n]}}\left|b_{r l}\right|, \widehat{v}=\max _{\mathfrak{L} \in[1, n]} v_{r}$, and $\widehat{\omega}=\max _{r \in[1, n]} \omega_{r}$. Define $\widetilde{L}=\left(\bar{l}_{i j}\right)_{N \times N}$ and $\widetilde{D}=\left(d_{i j}\right)_{N \times N}$, $i, j=1,2, \ldots, N$, where

$$
\begin{gathered}
\tilde{d}_{i j}= \begin{cases}2 d_{i j}^{(2 / 3)}, & i \neq j, \\
0, & i=j,\end{cases} \\
\tilde{l}_{i j}= \begin{cases}-\tilde{d}_{i j}, & i \neq j, \\
\sum_{j=1, j \neq i}^{N} \tilde{d}_{i j}, & i=j,\end{cases} \\
\tilde{\Lambda}=\operatorname{diag}\left\{\left(2 \tilde{\beta}_{1}\right)^{(2 / 3)},\left(2 \tilde{\beta}_{2}\right)^{(2 / 3)}, \ldots,\left(2 \tilde{\beta}_{N}\right)^{(2 / 3)}\right\} .
\end{gathered}
$$


The controller $u_{i}(t)$ is designed as follows:

$$
\begin{aligned}
u_{i}(t)= & -\Gamma\left\|e_{i}(t-\tau)\right\|_{1}^{2} \operatorname{sign}\left(e_{i}(t)\right) \\
& +\beta \sum_{j=1}^{N} d_{i j} \operatorname{sig}^{2}\left(e_{j}(t)-e_{i}(t)\right)-\beta \tilde{\beta} \operatorname{sig}^{2}\left(e_{i}(t)\right) \\
& -\operatorname{asign}\left(e_{i}(t)\right)-K \frac{e_{i}(t)}{\left\|e_{i}(t)\right\|^{2}} \\
& +\widehat{K} \sum_{l=1}^{n}\left(\int_{t-\tau}^{t} e_{i l}^{2}(s) \mathrm{d} s\right)^{(3 / 2)} \frac{e_{i}(t)}{\left\|e_{i}(t)\right\|^{2}},
\end{aligned}
$$

where $\quad \Gamma>0, \quad \quad \beta>0, \quad \operatorname{sign}\left(e_{i}(t)\right)=\operatorname{diag}$ $\left\{\operatorname{sign}\left(e_{i 1}(t)\right), \ldots, \operatorname{sign}\left(e_{\text {in }}(t)\right)\right\}, \quad a>0, \quad K>0, \quad \widehat{K}>0$, $\beta=\operatorname{diag}\left\{\widetilde{\beta}_{1}, \ldots, \widetilde{\beta}_{N}\right\}$. There exists a path between network $i$ and (6), if and only if, $\widetilde{\beta}_{i}>0$.

Note that controller (20) is discontinuous, we have

$$
\begin{aligned}
\overline{c o}\left[u_{i}(t)\right]= & -\Gamma\left\|e_{i}(t-\tau)\right\|_{1}^{2} \overline{c o}\left[\operatorname{sign}\left(e_{i}(t)\right)\right] \\
& +\beta \sum_{j=1}^{N} d_{i j} \overline{c o}\left[\operatorname{sig}^{2}\left(e_{j}(t)-e_{i}(t)\right)\right] \\
& -\beta \widetilde{\beta} \overline{c o}\left[\operatorname{sig}^{2}\left(e_{i}(t)\right)\right]-a \overline{c o}\left[\operatorname{sign}\left(e_{i}(t)\right)\right] \\
& -K \frac{e_{i}(t)}{\left\|e_{i}(t)\right\|^{2}} \\
& +\widehat{K} \sum_{l=1}^{n}\left(\int_{t-\tau}^{t} e_{i l}^{2}(s) \mathrm{d} s\right)^{(3 / 2)} \frac{e_{i}(t)}{\left\|e_{i}(t)\right\|^{2}},
\end{aligned}
$$

where $\quad e_{i}(t)>0, \quad \overline{c o}\left[\operatorname{sign}\left(e_{i}(t)\right)\right]=1 ; \quad e_{i}(t)=0$, $\overline{c o}\left[\operatorname{sign}\left(e_{i}(t)\right)\right]=[-1,1] ; e_{i}(t)<0, \overline{c o}\left[\operatorname{sign}\left(e_{i}(t)\right)\right]=-1$.

Let $\xi_{i}(t) \in \overline{c o}\left[u_{i}(t)\right]$, then there exists $\operatorname{Sign}\left(e_{i}(t)\right) \in \overline{c o}\left[\operatorname{sign}\left(e_{i}(t)\right)\right]$, such that

$$
\begin{aligned}
\xi_{i}(t)= & -\Gamma\left\|e_{i}(t-\tau)\right\|_{1}^{2} \operatorname{Sign}\left(e_{i}(t)\right) \\
& +\beta \sum_{j=1}^{N} d_{i j}\left|e_{j}(t)-e_{i}(t)\right|^{2} \operatorname{Sign}\left(e_{j}(t)-e_{i}(t)\right) \\
& -\beta \widetilde{\beta}\left|e_{i}(t)\right|^{2} \operatorname{Sign}\left(e_{i}(t)\right) \\
& +\widehat{K} \sum_{l=1}^{n}\left(\int_{t-\tau}^{t} e_{i l}^{2}(s) \mathrm{d}\right)^{(3 / 2)} \frac{e_{i}(t)}{\left\|e_{i}(t)\right\|^{2}} \\
& -a \operatorname{Sign}\left(e_{i}(t)\right)-K \frac{e_{i}(t)}{\left\|e_{i}(t)\right\|^{2}} .
\end{aligned}
$$

Theorem 1. Suppose that $\left(A_{1}\right),\left(A_{2}\right)$, and $\left(A_{3}\right)$ are satisfied, and the coupling interaction topology is undirected and connected. If the following conditions

$$
\begin{gathered}
\Theta=\left(\begin{array}{ll}
\Phi_{11} & \Phi_{12} \\
\Phi_{21} & \Phi_{22}
\end{array}\right)<0, \\
a-\Delta_{\max }-n \widehat{\omega} \widehat{b}-n \widehat{\omega} \widehat{a}>0, \\
\Gamma-\widehat{g} \widehat{b}>0, \\
\chi_{2}-2 n \widehat{g} \hat{a}>0,
\end{gathered}
$$

hold, where $\Phi_{11}=-2\left(I_{N} \otimes Q\right)+(2 n \hat{v} \hat{a}+1) \quad\left(I_{n N}\right)-$ $2 c_{1}\left(L \otimes I_{n}\right)+c_{2}\left(H \otimes I_{n}\right)\left(H \otimes I_{n}\right)^{T}, \quad \Phi_{12}=\widehat{\Phi_{21}}=\widehat{v} \widehat{b} \otimes I_{n}$, $\Phi_{21}=\widehat{v} \widehat{b} \otimes I_{n}, \Phi_{22}=\left(c_{2}-1\right) \otimes I_{n}, \chi_{2}=\left(\lambda_{\min }(\widetilde{L}+\widetilde{\Lambda})\right)^{(3 / 2)} \beta \widetilde{n}$ $(-1 / 2), \widehat{K}=\chi_{2}-2 n \hat{g} \widehat{a}, \widetilde{n}=n N^{2}$, then CNNs (1) are globally robust fixed-time synchronized with system (6) under the designed controller (20). And the upper bound $T_{\max }$ of settling time is estimated by

$$
T_{\max }=\frac{3}{2 K}\left(\frac{\widehat{K}}{2 K}\right)^{(2 / 3)}
$$

Proof. Construct the Lyapunov-Krasovskii functional

$$
\mathscr{V}(t)=\sum_{i=1}^{N} \sum_{l=1}^{n} \int_{t-\tau}^{t} e_{i l}^{2}(s) \mathrm{d} s+\sum_{i=1}^{N} e_{i}^{T}(t) e_{i}(t) .
$$

Calculating the derivative of $\mathscr{V}(t)$ at time $t$ along the trajectories of error system (9), it follows that

$$
\begin{aligned}
\dot{\mathscr{V}}(t)= & \sum_{i=1}^{N} 2 e_{i}^{T}(t) \dot{e}_{i}(t)-\sum_{i=1}^{N} e_{i}^{T}(t-\tau) e_{i}(t-\tau)+\sum_{i=1}^{N} e_{i}^{T}(t) e_{i}(t) \\
= & \sum_{i=1}^{N} 2 e_{i}^{T}(t)\left(-Q e_{i}(t)+A \tilde{f}\left(e_{i}(t)\right)+B \tilde{f}\left(e_{i}(t-\tau)\right)\right) \\
& +c_{1} \sum_{j=1}^{N} d_{i j} e_{j}(t)+\Delta_{i}\left(t, x_{i}(t)\right)+c_{2} \sum_{j=1}^{N} h_{i j} e_{j}(t-\tau) \\
& +u_{i}(t)+\sum_{i=1}^{N} e_{i}^{T}(t) e_{i}(t)-\sum_{i=1}^{N} e_{i}^{T}(t-\tau) e_{i}(t-\tau) \\
= & 2 \sum_{i=1}^{N} e_{i}^{T}(t) A \widehat{\gamma}_{i}(t)-2 \sum_{i=1}^{N} e_{i}^{T}(t) Q e_{i}(t) \\
& +2 \sum_{i=1}^{N} e_{i}^{T}(t) B \widehat{\gamma}_{i}(t-\tau)+2 \sum_{i=1}^{N} e_{i}^{T}(t) \Delta_{i}\left(t, x_{i}(t)\right) \\
& +2 c_{2} \sum_{i=1}^{N} \sum_{j=1}^{N} h_{i j} e_{i}^{T}(t)\left(e_{j}(t-\tau)-e_{i}(t-\tau)\right) \\
& +2 c_{1} \sum_{i=1}^{N} \sum_{j=1}^{N} d_{i j} e_{i}^{T}(t) e_{j}(t)+2 \sum_{i=1}^{N} e_{i}^{T}(t) u_{i}(t) \\
& -\sum_{i=1}^{N} e_{i}^{T}(t-\tau) e_{i}(t-\tau)+\sum_{i=1}^{N} e_{i}^{T}(t) e_{i}(t) .
\end{aligned}
$$

Substituting (22) into (31), we can obtain 


$$
\begin{aligned}
\dot{\mathscr{V}}(t)= & -2 \sum_{i=1}^{N} e_{i}^{T}(t) Q e_{i}(t)+2 \sum_{i=1}^{N} e_{i}^{T}(t) A \widehat{\gamma}_{i}(t)+\sum_{i=1}^{N} e_{i}^{T}(t) e_{i}(t) \\
& +2 \sum_{i=1}^{N} e_{i}^{T}(t) B \widehat{\gamma}_{i}(t-\tau)+2 c_{1} \sum_{i=1}^{N} \sum_{j=1}^{N} d_{i j} e_{i}^{T}(t)\left(e_{j}(t)-e_{i}(t)\right) \\
& +2 c_{2} \sum_{i=1}^{N} \sum_{j=1}^{N} h_{i j} e_{i}^{T}(t)\left(e_{j}(t-\tau)-e_{i}(t-\tau)\right) \\
& -2 \sum_{i=1}^{N} e_{i}^{T}(t) \Gamma\left\|e_{i}(t-\tau)\right\|_{1}^{2} \operatorname{Sign}\left(e_{i}(t)\right)-\sum_{i=1}^{N} e_{i}^{T}(t-\tau) e_{i}(t-\tau) \\
& -2 a \sum_{i=1}^{N} e_{i}^{T}(t) \operatorname{Sign}\left(e_{i}(t)\right)+2 \sum_{i=1}^{N} e_{i}^{T}(t) \\
& \cdot \beta \sum_{j=1}^{N} d_{i j}\left|e_{j}(t)-e_{i}(t)\right|^{2} \cdot \operatorname{Sign}\left(e_{j}(t)-e_{i}(t)\right) \\
& +2 \sum_{i=1}^{N} e_{i}^{T}(t) \Delta_{i}\left(t, x_{i}(t)\right)-2 \beta \tilde{\beta} \sum_{i=1}^{N} e_{i}^{T}(t)\left|e_{i}(t)\right|^{2} \operatorname{Sign}\left(e_{i}(t)\right) \\
& -2 K \sum_{i=1}^{N} e_{i}^{T}(t) \frac{e_{i}(t)}{\left\|e_{i}(t)\right\|^{2}}+2 \widehat{K} \sum_{i=1}^{N} e_{i}^{T}(t) \\
& \cdot \sum_{l=1}^{n}\left(\int_{t-\tau}^{t} e_{i l}^{2}(s) \mathrm{d} s\right)^{(3 / 2)} \frac{e_{i}(t)}{\left\|e_{i}(t)\right\|^{2}}
\end{aligned}
$$

Due to $d_{i j}=d_{j i}$, it follows that

$$
\begin{aligned}
\sum_{i=1}^{N} \sum_{j=1}^{N} d_{i j} e_{i}^{T}(t)\left(e_{j}(t)-e_{i}(t)\right) & \\
= & \frac{1}{2} \sum_{i=1}^{N} \sum_{j=1}^{N} d_{i j} e_{i}^{T}(t)\left(e_{j}(t)-e_{i}(t)\right) \\
& +\frac{1}{2} \sum_{i=1}^{N} \sum_{j=1}^{N} d_{j i} e_{j}^{T}(t)\left(e_{i}(t)-e_{j}(t)\right) \\
= & \frac{1}{2} \sum_{i=1}^{N} \sum_{j=1}^{N} d_{i j} e_{i}^{T}(t)\left(e_{j}(t)-e_{i}(t)\right) \\
& -\frac{1}{2} \sum_{i=1}^{N} \sum_{j=1}^{N} d_{i j} e_{j}^{T}(t)\left(e_{j}(t)-e_{i}(t)\right) \\
= & -\frac{1}{2} \sum_{i=1}^{N} \sum_{j=1}^{N} d_{i j}\left(e_{j}(t)-e_{i}(t)\right)^{T}\left(e_{j}(t)-e_{i}(t)\right) \\
= & -\frac{1}{2} \sum_{i=1}^{N} \sum_{j=1}^{N} \sum_{l=1}^{n} d_{i j}\left(e_{j l}(t)-e_{i l}(t)\right)^{2} \\
= & -e^{T}(t)\left(L \otimes I_{n}\right) e(t) .
\end{aligned}
$$

It is easy to derive that

$$
\begin{aligned}
2 c_{2} & \sum_{i=1}^{N} \sum_{j=1}^{N} h_{i j} e_{i}^{T}(t)\left(e_{j}(t-\tau)-e_{i}(t-\tau)\right) \\
& =2 c_{2} e^{T}(t)\left(H \otimes I_{n}\right) e(t-\tau) \\
& \leq c_{2} e^{T}(t)\left(H \otimes I_{n}\right)\left(H \otimes I_{n}\right)^{T} e(t)+c_{2} e^{T}(t-\tau) e(t-\tau)
\end{aligned}
$$

Similar to (31), one obtains

$$
\begin{aligned}
\sum_{i=1}^{N} \sum_{j=1}^{N} d_{i j} e_{i}^{T}(t)\left|e_{j}(t)-e_{i}(t)\right|^{2} \operatorname{Sign}\left(e_{j}(t)-e_{i}(t)\right) \\
=\frac{1}{2} \sum_{i=1}^{N} \sum_{j=1}^{N} d_{i j} e_{i}^{T}(t)\left|e_{j}(t)-e_{i}(t)\right|^{2} \operatorname{Sign}\left(e_{j}(t)-e_{i}(t)\right) \\
\quad+\frac{1}{2} \sum_{i=1}^{N} \sum_{j=1}^{N} d_{j i} e_{j}^{T}(t)\left|e_{j}(t)-e_{i}(t)\right|^{2} \operatorname{Sign}\left(e_{i}(t)-e_{j}(t)\right) \\
=\frac{1}{2} \sum_{i=1}^{N} \sum_{j=1}^{N} d_{j i} e_{i}^{T}(t)\left|e_{j}(t)-e_{i}(t)\right|^{2} \operatorname{Sign}\left(e_{j}(t)-e_{i}(t)\right)
\end{aligned}
$$

$$
\begin{aligned}
& -\frac{1}{2} \sum_{i=1}^{N} \sum_{j=1}^{N} d_{j i} e_{j}^{T}(t)\left|e_{j}(t)-e_{i}(t)\right|^{2} \operatorname{Sign}\left(e_{j}(t)-e_{i}(t)\right) \\
& =-\frac{1}{2} \sum_{i=1}^{N} \sum_{j=1}^{N} \sum_{l=1}^{n} d_{i j}\left|e_{j l}(t)-e_{i l}(t)\right|^{3} .
\end{aligned}
$$

By means of Assumptions $\left(A_{1}\right),\left(A_{2}\right)$, and $\left(A_{3}\right)$, we get

$$
\begin{aligned}
\sum_{i=1}^{N} e_{i}^{T}(t) A \widehat{\gamma}_{i}(t) & =\sum_{i=1}^{N} \sum_{r=1}^{n} \sum_{l=1}^{n} e_{i l}(t) a_{l r} \tilde{\gamma}_{i r}(t) \\
& \leq \sum_{i=1}^{N} \sum_{r=1}^{n} \sum_{l=1}^{n}\left|e_{i l}(t)\left\|a_{l r}\right\| \tilde{\gamma}_{i r}(t)\right| \\
& \leq \sum_{i=1}^{N} \sum_{r=1}^{n} \sum_{l=1}^{n}\left|e_{i l}(t)\right|\left|a_{l r}\right|\left(g_{r}\left|e_{i r}(t)\right|^{2}+v_{r}\left|e_{i r}(t)\right|+\omega_{r}\right) \\
& \leq n \widehat{v} \hat{a} \sum_{i=1}^{N} e_{i}^{T}(t) e_{i}(t)+n \widehat{g} \hat{a} \sum_{i=1}^{N} e_{i}^{T}(t) e_{i}^{2}(t)+n \widehat{\omega} \widehat{a} \sum_{i=1}^{N} \sum_{l=1}^{n}\left|e_{i l}(t)\right| .
\end{aligned}
$$

By Assumption $\left(\mathrm{A}_{2}\right)$, one has 


$$
\begin{aligned}
& \sum_{i=1}^{N} e_{i}^{T}(t) B \widehat{\gamma}_{i}(t-\tau) \\
& =\sum_{i=1}^{N} \sum_{r=1}^{n} \sum_{l=1}^{n} e_{i l}(t) b_{l r} \widetilde{\gamma}_{i r}(t-\tau) \\
& \leq \widehat{b} \sum_{i=1}^{N} \sum_{r=1}^{n} \sum_{l=1}^{n}\left|e_{i l}(t)\right|\left(\widetilde{\gamma}_{i r}(t-\tau)\right) \\
& \leq \widehat{b} \sum_{i=1}^{N} \sum_{r=1}^{n} \sum_{l=1}^{n}\left|e_{i l}(t)\right|\left(g_{r}\left|e_{i r}(t-\tau)\right|^{2}+v_{r}\left|e_{i r}(t-\tau)\right|+\omega_{r}\right) \\
& \leq \widehat{g} \widehat{b} \sum_{i=1}^{N}\left|e_{i}(t)\right|^{T}\left|e_{i}^{2}(t-\tau)\right|+\widehat{v} \widehat{b} \sum_{i=1}^{N}\left|e_{i}(t)\right|^{T}\left|e_{i}(t-\tau)\right| \\
& \quad+n \widehat{\omega} \widehat{b} \sum_{i=1}^{N} \sum_{l=1}^{n}\left|e_{i l}(t)\right| .
\end{aligned}
$$

Under Assumption $\left(\mathrm{A}_{3}\right)$, combining (30) with (35), it follows that

$$
\begin{aligned}
& \dot{\mathscr{V}}(t) \leq-2 e^{T}(t)\left(I_{N} \otimes Q\right) e(t) \\
& +2 n \widehat{v} \widehat{a} \sum_{i=1}^{N} e_{i}^{T}(t) e_{i}(t) 2 n \widehat{g} \widehat{a} \sum_{i=1}^{N} e_{i}^{T}(t) e_{i}^{2}(t) \\
& +2 n \widehat{\omega} \widehat{a} \sum_{i=1}^{N} \sum_{l=1}^{n}\left|e_{i l}(t)\right|+2 n \widehat{\omega} \widehat{b} \sum_{i=1}^{N} \sum_{l=1}^{n}\left|e_{i l}(t)\right| \\
& +2 \widehat{v} \widehat{b} \sum_{i=1}^{N} \sum_{l=1}^{n}\left|e_{i}^{T}(t)\right|\left|e_{i}(t-\tau)\right| \\
& -2 c_{1} e^{T}(t)\left(L \otimes I_{n}\right) e(t)+2 \hat{g} \widehat{b} \sum_{i=1}^{N}\left\|e_{i}(t)\right\|_{1}\left\|e_{i}(t-\tau)\right\|_{1}^{2} \\
& +c_{2} e^{T}(t)\left(H \otimes I_{n}\right)\left(H \otimes I_{n}\right)^{T} e(t)+c_{2} e^{T}(t-\tau) e(t-\tau) \\
& -2 \sum_{i=1}^{N} \sum_{l=1}^{n} e_{i}^{T}(t) \chi_{2}\left(\int_{t-\tau}^{t} e_{i l}^{2}(s) \mathrm{d} s\right)^{(3 / 2)} \frac{e_{i}(t)}{\left\|e_{i}(t)\right\|^{2}} \\
& +2 \Delta_{\max } \sum_{i=1}^{N} \sum_{l=1}^{n}\left|e_{i l}(t)\right| \\
& -2 \beta \sum_{i=1}^{N} \widetilde{\beta}_{i} \sum_{l=1}^{n}\left|e_{i l}(t)\right|^{3}-2 \sum_{i=1}^{N} e_{i}^{T}(t) K \frac{e_{i}(t)}{\left\|e_{i}(t)\right\|} \\
& -\sum_{i=1}^{N} e_{i}^{T}(t-\tau) e_{i}(t-\tau) \\
& +\sum_{i=1}^{N} e_{i}^{T}(t) e_{i}(t)-2 \Gamma \sum_{i=1}^{N}\left\|e_{i}^{T}(t)\right\|_{1}\left\|e_{i}(t-\tau)\right\|_{1}^{2} \\
& -\beta \sum_{i=1}^{N} \sum_{j=1}^{N} \sum_{l=1}^{n} d_{i j}\left|e_{j l}(t)-e_{i l}(t)\right|^{3}-2 a \sum_{i=1}^{N} \sum_{l=1}^{n}\left|e_{i l}(t)\right| \\
& +2 n \widehat{\operatorname{g}} \widehat{a} \sum_{i=1}^{N} \sum_{l=1}^{n} e_{i}^{T}(t)\left(\int_{t-\tau}^{t} e_{i l}^{2}(s) \mathrm{d} s\right)^{(3 / 2)} \text {. }
\end{aligned}
$$

The inequality above can be rewritten as

$$
\begin{aligned}
\dot{\mathscr{V}}(t) \leq & \eta \Theta \eta^{T}-\left(-2 n \widehat{\omega} \widehat{a}-2 n \widehat{\omega} \widehat{b}+2 a-2 \Delta_{\max }\right) \sum_{i=1}^{N} \sum_{l=1}^{n}\left|e_{i l}(t)\right| \\
& +2 n \widehat{g} \widehat{a} \sum_{i=1}^{N} \sum_{l=1}^{n} e_{i}^{T}(t)\left(\int_{t-\tau}^{t} e_{i l}^{2}(s) \mathrm{d} s\right)^{(3 / 2)} \\
& +2 n \widehat{g} \widehat{a} \sum_{i=1}^{N} e_{i}^{T}(t) e_{i}^{2}(t) \\
& -(-2 \widehat{g} \widehat{b}+2 \Gamma) \sum_{i=1}^{N}\left\|e_{i}(t)\right\|\left\|e_{i}(t-\tau)\right\|^{2} \\
& -2 \chi_{2} \sum_{i=1}^{N} \sum_{l=1}^{n} e_{i}^{T}(t)\left(\int_{t-\tau}^{t} e_{i l}^{2}(s) \mathrm{d} s\right)^{(3 / 2)} \\
& -\beta \sum_{i=1}^{N} \sum_{j=1}^{N} \sum_{l=1}^{n} d_{i j}\left|e_{j l}(t)-e_{i l}(t)\right|^{3} \\
& -2 \beta \sum_{i=1}^{N} \widetilde{\beta}_{i} \sum_{l=1}^{n}\left|e_{i l}(t)\right|^{3}-2 K,
\end{aligned}
$$

where $\eta=\left[e_{i}(t), e_{i}(t-\tau)\right]^{T}$.

Together (24)-(26) and (37), one has

$$
\begin{aligned}
\dot{\mathscr{V}}(t) \leq & 2 n \hat{\mathrm{g}} \widehat{a} \sum_{i=1}^{N} e_{i}^{T}(t) e_{i}^{2}(t) \\
& +2 n \hat{g} \hat{a} \sum_{i=1}^{N} \sum_{l=1}^{n} e_{i}^{T}(t)\left(\int_{t-\tau}^{t} e_{i l}^{2}(s) \mathrm{d} s\right)^{(3 / 2)} \\
& -\beta \sum_{i=1}^{N} \sum_{j=1}^{N} \sum_{l=1}^{n} d_{i j}\left|e_{j l}(t)-e_{i l}(t)\right|^{3} \\
& -2 \beta \sum_{i=1}^{N} \widetilde{\beta}_{i} \sum_{l=1}^{n}\left|e_{i l}(t)\right|^{3}-2 K \\
& -2 \chi_{2} \sum_{i=1}^{N} \sum_{l=1}^{n} e_{i}^{T}(t)\left(\int_{t-\tau}^{t} e_{i l}^{2}(s) \mathrm{d} s\right)^{(3 / 2)} .
\end{aligned}
$$

Using Lemma 4 yields

$$
\sum_{i=1}^{N} \sum_{l=1}^{n}\left|e_{i l}(t)\right| \geq\left(\sum_{i=1}^{N} \sum_{l=1}^{n}\left|e_{i l}(t)\right|^{2}\right)^{(1 / 2)}=\left(e^{T}(t) e(t)\right)^{(1 / 2)} \text {. }
$$


By Lemma 3 and (18), one has

$$
\begin{aligned}
& -\beta \sum_{i=1}^{N} \sum_{j=1}^{N} \sum_{l=1}^{n} d_{i j}\left|e_{j l}(t)-e_{i l}(t)\right|^{3}-2 \beta \sum_{i=1}^{N} \tilde{\beta}_{i} \sum_{l=1}^{n}\left|e_{i l}(t)\right|^{3} \\
& \leq-\beta \bar{n}^{-(1 / 2)}\left(\sum_{l=1}^{n}\left(\sum_{j=1}^{N} \sum_{i=1}^{N} d_{i j}^{(2 / 3)}\left|e_{j l}(t)-e_{i l}(t)\right|^{2}+\sum_{i=1}^{N}\left(2 \tilde{\beta}_{i}\right)^{(2 / 3)}\left|e_{i l}(t)\right|^{2}\right)\right)^{(3 / 2)} \\
& =-\beta \bar{n}^{-(1 / 2)}\left(\sum_{i=1}^{N} \sum_{j=1}^{N} \sum_{l=1}^{n} \frac{1}{2} \widetilde{d}_{i j}\left(e_{j l}(t)-e_{i l}(t)\right)^{2}+\sum_{l=1}^{n} \sum_{i=1}^{N}\left(2 \widetilde{\beta}_{i}\right)^{(2 / 3)}\left|e_{i l}(t)\right|^{2}\right)^{(3 / 2)} \\
& =-\beta \bar{n}^{-(1 / 2)}\left(e^{T}(t)(\widetilde{L} \otimes I) e(t)+e^{T}(t)(\tilde{\wedge} \otimes I) e(t)\right)^{(3 / 2)}=-\tilde{\beta n}^{-(1 / 2)}\left(e^{T}(t)((\tilde{\wedge}+\widetilde{L}) \otimes I) e(t)\right)^{(3 / 2)}
\end{aligned}
$$

where $m$ is the real number nodes of $\widetilde{\beta}_{i}$, $\bar{n}=n(N(N-1)+m), \quad$ and $\quad m_{\max }=N$. Then, $\tilde{n}=n(N(N-1)+m)=n N^{2} \geq \bar{n}$. Combining (39) with (40), we have

$$
\dot{\mathscr{V}}(t) \leq-\left(-2 n \hat{g} \hat{a}+\beta \tilde{n}^{(-1 / 2)}\left(\lambda_{\min }(\tilde{\wedge}+\widetilde{L}) \otimes I\right)^{(3 / 2)}\right) \mathscr{V}^{(3 / 2)}-2 K .
$$

Noting that $\tilde{\wedge}+\widetilde{L}$ is positive definite, we can get

$$
\dot{\mathscr{V}}(t) \leq-\left(\widehat{K} \mathscr{V}^{(3 / 2)}(t)+2 K\right)
$$

where $\widehat{K}=-2 n \hat{g} \widehat{a}+\beta \widetilde{n}^{(-1 / 2)}\left(\lambda_{\min }(\widetilde{\wedge}+\widetilde{L}) \otimes I\right)^{(3 / 2)}>0$.

By Lemma 3, we can conclude that error system (9) is globally robust fixed-time stable. This shows that system (1) can achieve the global robust fixed-time synchronization with system (6) under the controller (20). The upper bound $T_{\max }$ of settling time is estimated by $T_{\max }=(3 / 2 K)(\widehat{K} / 2 K)^{(2 / 3)}$. The proof is completed.

Remark 1. In [30, 33-36], the global fixed-time synchronization issues were considered for delayed CNNs with discontinuous activations, where activation function $f(\cdot)$ is subject to linear growth. However, in Theorem 1, discontinuous activations $f(\cdot)$ are nonlinear growth and subject to a quadratic polynomial function. In addition, in [37], the Lyapunov function $\mathscr{V}(t)=\sum_{i=1}^{N} e_{i}^{T}(t) e_{i}(t)$ is used to achieve the global fixed-time synchronization conditions. In this paper, the integral item $\sum_{i=1}^{N} \sum_{l=1}^{n} \int_{t-\tau}^{t} e_{i l}^{2}(s) \mathrm{d} s$ is introduced in Lyapunov functional (28). Obviously, compared with the above works in $[30,33-36]$, the result in this paper is more general.

Remark 2. It should be pointed out that the upper bound of the settling time, $T_{\max }=(3 / 2 K)(\widehat{K} / 2 K)^{(2 / 3)}$, is independent on initial conditions. In addition, it is easy to see that, on the basis of the configuration for parameters $K$ in the proposed control law, the upper bound $T_{\max }$ can be determined in advance to any desired values.

In the designed controller (20), the integral item $\sum_{l=1}^{n}\left(\int_{t-\tau}^{t} e_{i l}^{2}(s) \mathrm{d} s\right)^{(3 / 2)}$ is used, which may bring difficulties in implement. We remove this integral item and design the following controller:

$$
\begin{aligned}
u_{i}(t)= & -\Gamma\left\|e_{i}(t-\tau)\right\|_{1}^{2} \operatorname{sign}\left(e_{i}(t)\right)+\beta \sum_{j=1}^{N} d_{i j} \operatorname{sig}^{2}\left(e_{j}(t)-e_{i}(t)\right)-\beta \tilde{\beta} \operatorname{sig}^{2}\left(e_{i}(t)\right) \\
& -\operatorname{asign}\left(e_{i}(t)\right)-K \frac{e_{i}(t)}{\left\|e_{i}(t)\right\|^{2}}-\Gamma_{2}\left\|e_{i}(t-\tau)\right\|_{1} \operatorname{sign}\left(e_{i}(t)\right) .
\end{aligned}
$$
result.

Applying controller (43), we can obtain the following

Corollary 1. Suppose that Assumptions $\left(A_{1}\right),\left(A_{2}\right)$, and $\left(A_{3}\right)$ are satisfied and couple interaction topology is connected and undirected. If the following conditions

$$
\begin{gathered}
\Theta_{1}=\left(\begin{array}{cc}
\Psi_{11} & \Psi_{12} \\
\Psi_{21} & \Psi_{22}
\end{array}\right)<0, \\
a-\Delta_{\max }-n \widehat{\omega} \widehat{b}-n \widehat{\omega} \widehat{a}>0, \\
\Gamma-\widehat{g} \widehat{b}>0,
\end{gathered}
$$


hold, where $\Psi_{11}=-2\left(I_{N} \otimes Q\right)+2 n \widehat{v} \widehat{a}\left(I_{n N}\right)-2 c_{1}\left(L \otimes I_{n}\right)+$ $c_{2}\left(H \otimes I_{n}\right)\left(H \otimes I_{n}\right)^{T}+\left(I_{N} \otimes I_{n}\right), \Psi_{12}=\widehat{\Phi_{21}}=n \widehat{v} \widehat{b} I_{n N}, \Psi_{21}=$ $n \widehat{v} \vec{b} \otimes I_{n}, \quad \Psi_{22}=c_{2} I_{n N}, \chi_{2}=\left(\lambda_{\min }(\widetilde{L}+\widetilde{\Lambda})\right)^{(3 / 2)} \quad \beta \widetilde{n}^{(-1 / 2)}$, $\tilde{n}=n N^{2}$, then CNNs (1) are globally robust fixed-time synchronized with system (6) under controller (43). Also, the settling time is estimated by

$$
T_{\max }=\frac{3}{2 \Gamma_{2}}\left(\frac{\chi_{2}}{2 \Gamma_{2}}\right)^{(2 / 3)}
$$

Proof. Consider $\mathscr{V}(t)=\sum_{i=1}^{N} e_{i}^{T}(t) e_{i}(t)$ and follow the similar proof process of Theorem 1 , and Corollary 1 can easily be proved, hence omitted here.

Next, we developed the global robust finite-time synchronization conditions for CNNs (1). Design the following controller:

$$
\begin{aligned}
u_{i}(t)= & -\Gamma\left\|e_{i}(t-\tau)\right\|_{1}^{2} \operatorname{sign}\left(e_{i}(t)\right)-\Pi\left\|e_{i}(t)\right\|_{1}^{2} \operatorname{sign}\left(e_{i}(t)\right) \\
& -\operatorname{asign}\left(e_{i}(t)\right)+\widehat{K} \sum_{l=1}^{n}\left(\int_{t-\tau}^{t} e_{i l}^{2}(s) \mathrm{d} s\right)^{(1 / 2)} \frac{e_{i}(t)}{\left\|e_{i}(t)\right\|^{2}},
\end{aligned}
$$

where $\quad \Gamma>0, \quad \Pi>0, \quad a>0, \quad \widehat{K}>0, \quad$ and $\operatorname{sign}\left(e_{i}(t)\right)=\operatorname{diag}\left\{\operatorname{sign}\left(e_{i 1}(t)\right), \ldots, \operatorname{sign}\left(e_{i n}(t)\right)\right\}$.

Theorem 2. Suppose that Assumptions $\left(A_{1}\right),\left(A_{2}\right)$, and $\left(A_{3}\right)$ are satisfied and couple interaction topology is connected and undirected. If the following conditions

$$
\begin{array}{r}
\Theta_{2}=\left(\begin{array}{cc}
\Phi_{11} & \Phi_{12} \\
\Phi_{21} & \Phi_{22}
\end{array}\right)<0, \\
\Pi-n \hat{g} \hat{a}>0, \\
2 \Gamma-2 \hat{g} \hat{b}>0,
\end{array}
$$

hold, $\quad$ where $\Phi_{11}=-2\left(I_{N} \otimes Q\right)+(2 n \hat{v} \widehat{a}+1) I_{n N}-$ $2 c_{1}\left(L \otimes I_{n}\right)+c_{2}\left(H \otimes I_{n}\right)\left(H \otimes I_{n}\right)^{T}, \Phi_{12}=\widehat{\Phi_{21}}=n \widehat{\nu} \widehat{b} \otimes I_{n}$, $\Phi_{21}=n \widehat{\nu} \widehat{b} \otimes I_{n}, \Phi_{22}=\left(c_{2}-1\right) I_{n N}, \chi_{1}=-n \widehat{\omega} \widehat{a}-n \widehat{\omega} \widehat{b}+a-$ $\Delta_{\text {max }}, \mathscr{V}(0)=\sum_{i=1}^{N}\left\|e_{i}(0)\right\|_{1}$, then CNNs (1) are globally robust finite-time synchronized under the controller (46). And the setting time is estimated by

$$
t_{1}\left(\widetilde{\varphi}_{i}(s), \widetilde{\phi}_{i}(s)\right)=\frac{2 V^{(1 / 2)}(0)}{\chi_{1}} .
$$

Proof. Consider Lyapunov-Krasovskii functional (28). Analogous as the proof of Theorem 1, we can get

$$
\begin{aligned}
\dot{\mathscr{V}}(t) \leq & -\left(-2 n \widehat{\omega} \widehat{a}-2 n \widehat{\omega} \widehat{b}+2 a-2 \Delta_{\max }\right) \sum_{i=1}^{N} \sum_{l=1}^{n}\left|e_{i l}(t)\right|+\eta \Theta_{2} \eta^{T} \\
& +2 \chi_{1} \sum_{i=1}^{N} \sum_{l=1}^{n} e_{i}^{T}(t)\left(\int_{t-\tau}^{t} e_{i l}^{2}(s) \mathrm{d} s\right)^{(1 / 2)} \\
& -(\Pi-2 n \widehat{g} \widehat{a}) \sum_{i=1}^{N}\left|e_{i}^{T}(t)\right|\left|e_{i}(t)\right|^{2} \\
& -(-2 \widehat{g} \widehat{b}+2 \Gamma) \sum_{i=1}^{N}\left\|e_{i}(t)\right\|\left\|e_{i}(t-\tau)\right\|^{2},
\end{aligned}
$$

where $\eta=\left[e_{i}(t), e_{i}(t-\tau)\right]^{T}$.

By (47)-(49) and (51), it follows that

$$
\begin{aligned}
\dot{\mathscr{V}}(t) \leq & 2 \chi_{1} \sum_{i=1}^{N} \sum_{l=1}^{n} e_{i}^{T}(t)\left(\int_{t-\tau}^{t} e_{i l}^{2}(s) \mathrm{d} s\right)^{(1 / 2)} \\
& -\left(-2 n \widehat{\omega} \widehat{a}-2 n \widehat{\omega} \widehat{b}+2 a-2 \Delta_{\max }\right) \sum_{i=1}^{N} \sum_{l=1}^{n}\left|e_{i l}(t)\right| .
\end{aligned}
$$

Furthermore, we have

$$
\dot{\mathscr{V}}(t) \leq \chi_{1} \mathscr{V}^{(1 / 2)}(t)
$$

Applying Lemma 2, we can conclude that error system (9) is globally robust finite-time stable. This shows that system (1) can achieve the global robust finite-time synchronization with system (6) under the controller (46). The settling time is estimated by $t_{1}\left(\widetilde{\varphi}_{i}(s), \widetilde{\phi}_{i}(s)\right)=\left(\left(2 \mathscr{V}^{(1 / 2)}(0)\right) / \chi_{1}\right)$. The proof is completed.

\section{Numerical Examples}

Example 1. Consider an array CNNs (1) with five NNs, in which the dynamical equation of each network is described by

$$
\begin{aligned}
\dot{x}_{i l}(t)= & -Q x_{i l}(t)+A f\left(x_{i l}(t)\right)+B f\left(x_{i l}(t-\tau)\right)+\Delta_{i l}\left(t, x_{i}(t)\right) \\
& +c_{1} \sum_{j=1}^{5} d_{i j}\left(x_{j l}(t)-x_{i l}(t)\right) \\
& +c_{2} \sum_{j=1}^{5} h_{i j}\left(x_{j l}(t-\tau)-x_{i l}(t-\tau)\right) \\
& +J(t)+u_{i}(t), \quad l=1,2, i=1, \ldots, 5 .
\end{aligned}
$$

The corresponding isolated network (6) is given by

$\dot{y}(t)=-Q y(t)+A f(y(t))+B f(y(t-\tau))+J(t)$, 


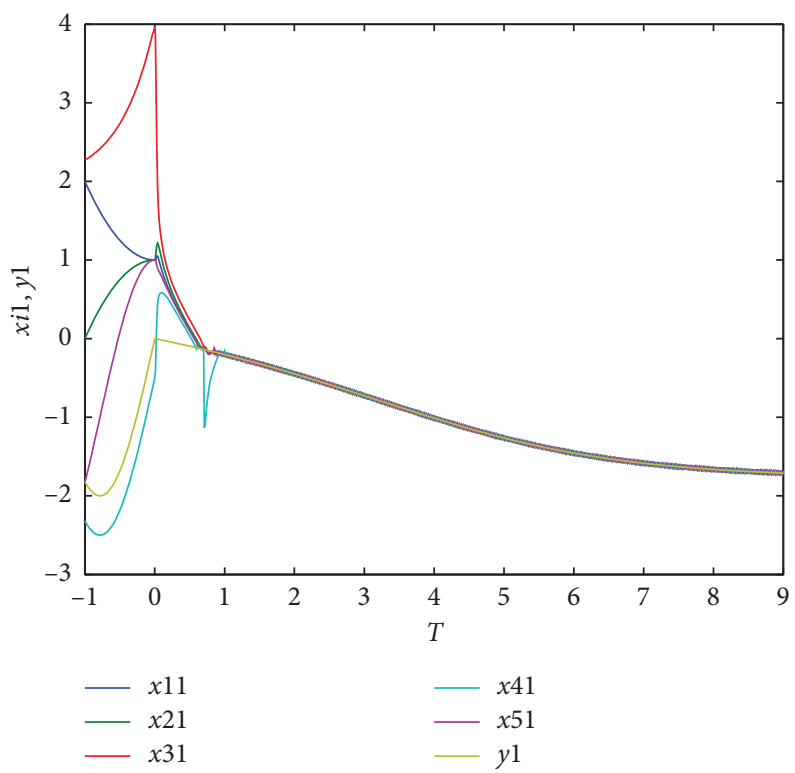

FIgURE 1: State trajectories of variables $x_{i 1}(t)$ and $y_{1}(t), i=1, \ldots, 5$.

where $\quad A=\left(\begin{array}{ll}0.12 & 0.3 \\ 0.44 & 0.5\end{array}\right), B=\left(\begin{array}{cc}0.2 & 0.3 \\ -0.36 & -0.06\end{array}\right), \quad$ and $Q=\left(\begin{array}{cc}5.51 & 0 \\ 0 & 6.91\end{array}\right) . \quad$ Take $\quad D=\left(\begin{array}{lllll}1 & 1 & 1 & 1 & 1 \\ 1 & 1 & 1 & 0 & 1 \\ 1 & 1 & 1 & 1 & 0 \\ 1 & 0 & 1 & 1 & 1 \\ 1 & 1 & 0 & 1 & 1\end{array}\right), \quad L=$ $\left(\begin{array}{ccccc}4 & -1 & -1 & -1 & -1 \\ -1 & 3 & -1 & 0 & -1 \\ -1 & -1 & 3 & -1 & 0 \\ -1 & 0 & -1 & 3 & -1 \\ -1 & -1 & 0 & -1 & 3\end{array}\right)$, and $H=\left(\begin{array}{ccccc}1 & -1 & 0 & -1 & -2 \\ -1 & 1 & -1 & -1 & 0 \\ 0 & -1 & 1 & 0 & 1 \\ -1 & -1 & 0 & 1 & -1 \\ -2 & 0 & -1 & -1 & 1\end{array}\right)$. The initial values are selected as $\widetilde{\varphi}_{1}(t)=\left(2 t^{2}+\right.$ $1,2 \cos 2 t+2)^{T}, \quad \tilde{\varphi}_{2}(t)=\left(-2 t^{2}+1,2 \sin 2 t+3\right)^{T}, \quad \tilde{\varphi}_{3}(t)=$ $\left(0.2 \sin 2 t+1,0.1 e^{3 t}+e^{t}\right)^{T}, \quad \tilde{\varphi}_{4}(t)=\left(2 \sin 2 t-0.5, e^{t}\right)^{T}, \quad$ and $\widetilde{\varphi}_{5}(t)=\left(2 \cos 2 t+1,0.1 e^{3 t}+e^{t}\right)^{T}$. The initial condition of the corresponding isolated network is $\widetilde{\psi}(t)=(2 \sin 2 t, 2 \sin t)^{T}$. Take activation function $f$ as $f\left(x_{i l}(t)\right)=0.001 x_{i l}^{2}(t)+$ $0.05 \operatorname{sign}\left(x_{i l}(t)\right), i=1,2, \ldots, 5, \quad$ where $\quad x_{i l}(t) \in \widehat{D}, \widehat{D}=$ $[-10,10] \times[-10,10]$, then $\widehat{g}=0.001, \widehat{v}=0, \widehat{\omega}=0.45$.

$$
\begin{array}{r}
\Delta\left(x_{i}(t)\right)=\left(0.7 \sin \left(x_{i 1}(t)\right), 0.6 \sin \left(x_{i 2}(t)\right)\right)^{T}, \\
i=1,2, \ldots, 5, \Delta_{\max }=\sqrt{0.85} .
\end{array}
$$

Set $\tau=1, \quad c_{1}=0.4, \quad c_{2}=1, \quad a=4.15, \quad \widetilde{\beta}=\operatorname{diag}\{3.14$, $5.19,0,0,0\}, K=0.25, \beta=1.412$, and $\Gamma=5.863$. By simple calculation, $\quad a-\Delta_{\max }-n \widehat{\omega} \widehat{b}-n \widehat{\omega} \widehat{a}=2.544>0, \quad \Gamma-\widehat{g} \widehat{b}=$ $\left.5.862>0, \quad \chi_{2}=\lambda_{\min }(\widetilde{L}+\widetilde{\Lambda})\right)^{(3 / 2)} \beta \widetilde{n}^{(-1 / 2)}=0.636$, and $\widehat{K}=$ $\chi_{2}-2 n \hat{g} \widehat{a}=0.6344>0$.

Then, we calculate $T_{\max }=7.032 \mathrm{~s}$. Moreover, it is easy to verify that condition (23) in Theorem 1 is satisfied. As shown in Figures 1-3, the simulation results agree well with the theoretical analysis. Specifically, Figures 1 and 2 depict the state variables of system (54) and (55). Figure 3 shows the evolutions of $e_{i j}, i, j=1,2, \ldots, 5$. From Figure 3 , it is easy to view that systems (54) and (55) can achieve the global robust synchronization in fixed time $T_{\max }=1.2 \mathrm{~s}$ under controller (20).

Example 2. Consider an array delayed CNNs (1) with three NNs, in which the dynamical equation of each network described by

$$
\begin{aligned}
\dot{x}_{i l}(t)= & -Q x_{i l}(t)+A f\left(x_{i l}(t)\right)+B f\left(x_{i l}(t-\tau)\right)+\Delta_{i l}\left(t, x_{i}(t)\right) \\
& +c_{1} \sum_{j=1}^{3} d_{i j}\left(x_{j l}(t)-x_{i l}(t)\right) \\
& +c_{2} \sum_{j=1}^{3} h_{i j}\left(x_{j l}(t-\tau)-x_{i l}(t-\tau)\right) \\
& +J(t)+u_{i}(t), \quad l=1,2,3, i=1,2,3 .
\end{aligned}
$$

The corresponding isolated network (6) is given by

$$
\dot{y}(t)=-Q y(t)+A f(y(t))+B f(y(t-\tau))+J(t),
$$

where $A=\left(\begin{array}{ccc}0.6 & 0.11 & -0.1 \\ 0.4 & 0.65 & 1 \\ 1 & 0.7 & 0.4\end{array}\right), B=\left(\begin{array}{ccc}0.2 & 0.3 & -0.19 \\ -0.26 & 0.06 & 0.03 \\ 0.3 & 0.1 & 0.1\end{array}\right)$, and $Q=\left(\begin{array}{ccc}5.1 & 0 & 0 \\ 0 & 2.2 & 0 \\ 0 & 0 & 2.01\end{array}\right)$. Take $D=\left(\begin{array}{ccc}-1 & 1 & 0 \\ 1 & -2 & 1 \\ 0 & 1 & 1\end{array}\right), \quad L=$ $\left(\begin{array}{ccc}1 & -1 & 0 \\ -1 & 2 & -1 \\ 0 & -1 & 1\end{array}\right)$, and $H=\left(\begin{array}{ccc}-1 & 1 & 0 \\ 1 & -2 & 1 \\ 0 & 1 & -1\end{array}\right)$. The initial values are selected as $\widetilde{\varphi}_{1}(t)=\left(2 t^{2}+1,2.1 \cos 2 t+\right.$ $\left.2,2.5 t^{2}+1\right)^{T}, \quad \widetilde{\varphi}_{2}(t)=\left(-2 t^{2}+1,2.5 t+3,2 \sin t+1\right)^{T}$, and $\widetilde{\varphi}_{3}(t)=\left(0.2 \sin 2 t+1,0.1 e^{3 t}+e^{t}, e^{3 t}\right)^{T}$. The trivial point $y(t)=0$ is taken as the objective trajectory. 


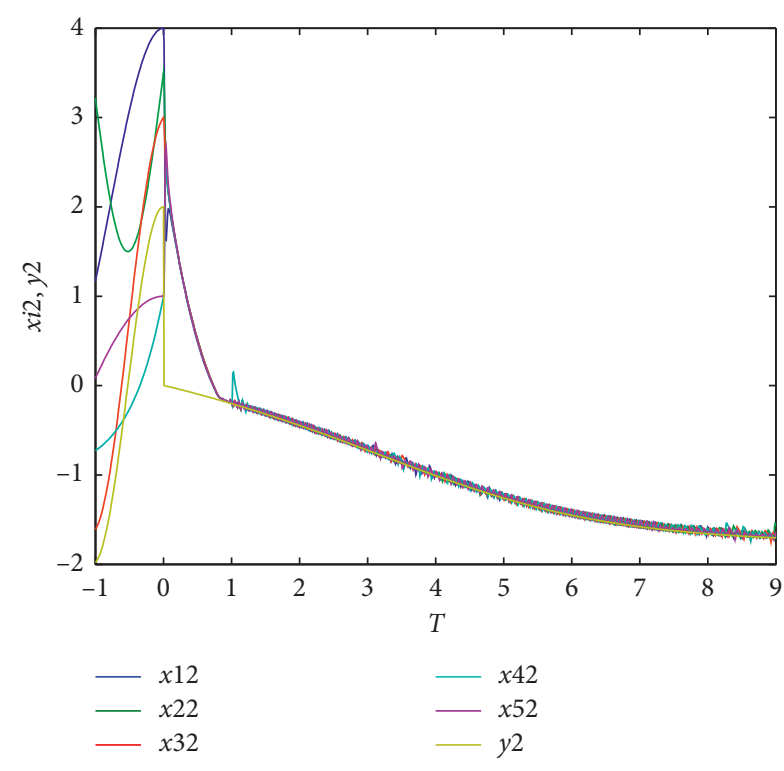

Figure 2: State trajectories of variables $x_{i 2}(t)$ and $y_{2}(t), i=1, \ldots, 5$.

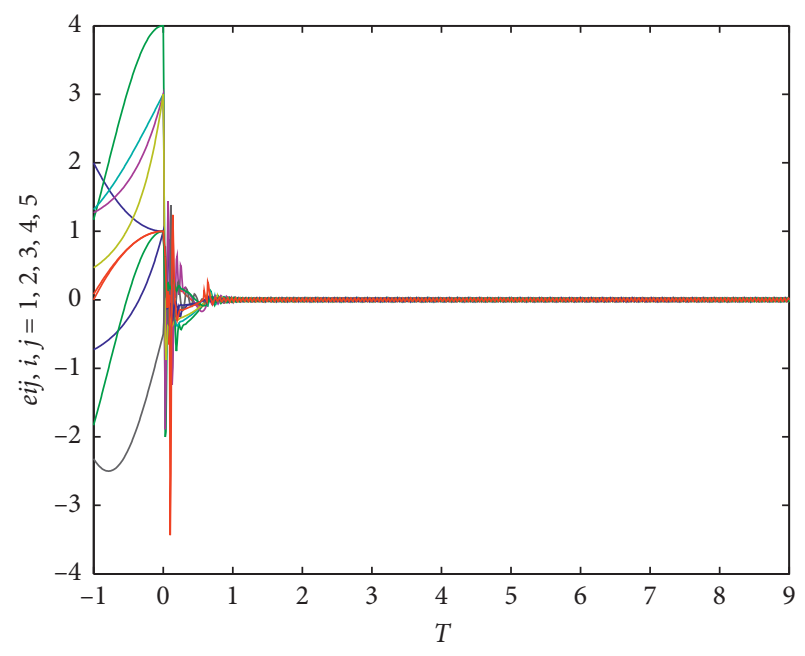

Figure 3: Time evolutions of $e_{i j}, i, j=1, \ldots, 5$ under the controller (20).

We take the activation functions $f$ as $f\left(x_{i l}(t)\right)=$ $0.01 x_{i l}^{2}(t)+0.3 \operatorname{sign}\left(x_{i l}(t)\right), \quad i=1,2,3$, where $x_{i l}(t) \in \widehat{D}$, $\widehat{D}=[-10,10] \times[-10,10]$, then $\widehat{g}=0.01, \widehat{v}=0, \widehat{\omega}=0.5$.

Let $\Delta\left(x_{i}(t)\right)=\left(0.2 \sin \left(x_{i 1} \quad(t)\right), 0.3 \sin \quad\left(x_{i 2}(t)\right)\right.$, $\left.0.4 \sin \left(x_{i 3}(t)\right)\right)^{T}, i=1,2,3, \quad \Delta_{\max }=\sqrt{0.29}$. Set $\tau=1$, $c_{1}=0.04, \quad c_{2}=1.8, \quad a=3.01, \quad \Pi=0.48, \quad \widehat{K}=1.121$, and $\Gamma=0.42$. By simple computation, $\Pi-n \hat{g} \hat{a}=0.45>0$, and $\Gamma-\hat{g} \widehat{b}=0.414>0$.

From (50), $\chi_{1}=-n \widehat{\omega} \widehat{a}-n \widehat{\omega} \widehat{b}+a-\Delta_{\max }=0.522$. Then, we calculate $t_{1}\left(\widetilde{\varphi}_{i}(s), \widetilde{\phi}_{i}(s)\right)=21.979 s$. Moreover, it is easy to verify that condition (50) in Theorem 2 also satisfied. As shown in Figure 4, the simulation results agree well with the theoretical analysis. Figure 4 shows the evolutions of $e_{i j}$, $i, j=1,2,3$, and it is obvious to view that systems (57) and

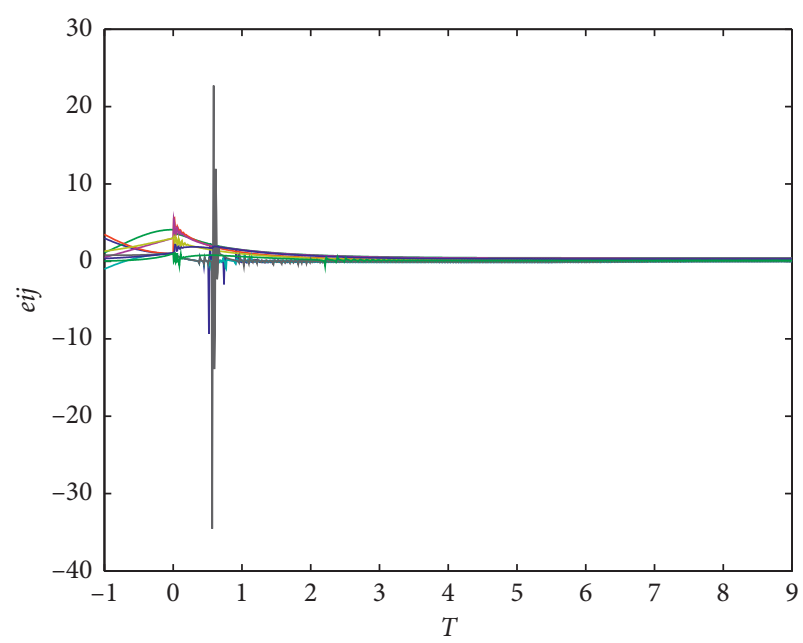

FIgURE 4: Time response of synchronization errors $\left\|e_{i}\right\|, i=1,2,3$ under the controller (46).

(58) can achieve THE global robust finite-time synchronization before $T=3.8 s$ under controller (46).

\section{Conclusion}

In this paper, the global robust synchronization in fixed time and global synchronization in finite time have been investigated for a class of hybrid coupled delayed NNs with discontinuous activation functions. Under the designed discontinuous feedback controller, the global synchronization conditions have been presented in the forms of LMIs, and the settling time, which is independent on initial conditions, has been also evaluated. Compared with the existing works, where the neuron activations are supposed to be linear growth, the results proposed in this paper are more general.

It is worth noting that the designed feedback controllers contain the sign function and the integral term, which may bring the chatter in actual implement, indicating that the designed control schemes have certain limitation.

Future work will be focused on how to remove the chatter of the designed controller and to extend the results here obtained for stochastic sampled-date synchronization control for CNNs with delays and discontinuous activations.

\section{Data Availability}

The underlying data supporting the results of our study can be found in the original paper.

\section{Conflicts of Interest}

The authors declare that they have no conflicts of interest.

\section{References}

[1] A. Bergman and M. L. Siegal, "Evolutionary capacitance as a general feature of complex gene networks," Nature, vol. 424, no. 6948, pp. 549-552, 2003. 
[2] Y. Jiang and J. Yang, "Complex dynamics in a food chain with slow and fast processes," Chaos, Solitons \& Fractals, vol. 42, no. 5, pp. 3160-3168, 2009.

[3] S. Liu and F. Zhang, "Complex function projective synchronization of complex chaotic system and its applications in secure communication," Nonlinear Dynamics, vol. 76, no. 2, pp. 1087-1097, 2014.

[4] D. M. Zhang, S. Yao, and X. W. Li, "Research and implementation of uplink synchronization in TD-LTE," Advanced Materials Research, vol. 759, pp. 846-850, 2013.

[5] R. Rakkiyappan, G. Velmurugan, and J. Cao, "Stability analysis of fractional-order complex-valued neural networks with time delays," Chaos, Solitons \& Fractals, vol. 78, pp. 297-316, 2015.

[6] Y. Tang, W. Wong, and W. K. Wong, "Distributed synchronization of coupled neural networks via randomly occurring control," IEEE Transactions on Neural Networks and Learning Systems, vol. 24, no. 3, pp. 435-447, 2013.

[7] J. Wang, H. Wu, and T. Huang, "Passivity and output synchronization of complex dynamical networks with fixed and adaptive coupling strength," IEEE Transactions on Neural Networks and Learning Systems, vol. 29, pp. 364-376, 2016.

[8] J. Zhang and Y. Gao, "Synchronization of coupled neural networks with time-varying delay," Neurocomputing, vol. 219, pp. 154-162, 2017.

[9] M. Xu, J.-L. Wang, and P.-C. Wei, "Synchronization for coupled reaction-diffusion neural networks with and without multiple time-varying delays via pinning-control," Neurocomputing, vol. 227, pp. 82-91, 2017.

[10] S.-X. Wang, Y.-L. Huang, and B.-B. Xu, "Pinning synchronization of spatial diffusion coupled reaction-diffusion neural networks with and without multiple time-varying delays," Neurocomputing, vol. 227, pp. 92-100, 2017.

[11] H. Zhang, Y. Sheng, and Z. Zeng, "Synchronization of Coupled Reaction-Diffusion neural networks with directed topology via an adaptive approach," IEEE Transactions on Neural Networks and Learning Systems, vol. 29, no. 5, pp. 1550-1561, 2018.

[12] H. Meng, N. Bianchi-Berthouze, Y. Deng, J. Cheng, and J. P. Cosmas, "Time-delay neural network for continuous emotional dimension prediction from facial expression sequences," IEEE Transactions on Cybernetics, vol. 46, no. 4, pp. 916-929, 2016.

[13] H. Shao and Z. Zhang, "Delay-dependent state feedback stabilization for a networked control model with two additive input delays," Applied Mathematics and Computation, vol. 265, pp. 748-758, 2015.

[14] W. He, F. Qian, and J. Cao, "Pinning-controlled synchronization of delayed neural networks with distributed-delay coupling via impulsive control," Neural Networks, vol. 85, pp. 1-9, 2017.

[15] J. Wang and S. Huang, "Pinning control for synchronization of coupled reaction-diffusion neural networks with directed topologies," IEEE Transactions on Systems, Man, and Cybernetics: Systems, vol. 46, pp. 1109-1120, 2017.

[16] Z. Cai and L. Huang, "Novel adaptive control and statefeedback control strategies to finite-time stabilization of discontinuous delayed networks," IEEE Transactions on Systems, Man, and Cybernetics: Systems, vol. 47, no. 7, pp. 1644-1654, 2017.

[17] L. Duan, L. Huang, and X. Fang, "Finite-time synchronization for recurrent neural networks with discontinuous activations and time-varying delays," Chaos: An Interdisciplinary Journal of Nonlinear Science, vol. 27, no. 1, p. 13101, 2017.
[18] X. Yang and J. Cao, "Exponential synchronization of delayed neural networks with discontinuous activations," IEEE Transactions on Circuits and Systems I: Regular Papers, vol. 60, no. 9, pp. 2431-2439, 2013.

[19] X. Peng, H. Wu, and J. Cao, "Global nonfragile synchronization in finite time for fractional-order discontinuous neural networks with nonlinear growth activations," IEEE Transactions on Neural Networks and Learning Systems, vol. 30, no. 7, pp. 2123-2137, 2019.

[20] V. T. Haimo, "Finite time controllers," SIAM Journal on Control and Optimization, vol. 24, no. 4, pp. 760-770, 1986.

[21] S. Bhat, "Finite-time stability of homogeneous systems," Proceedings of ACC, vol. 4, pp. 2513-2514, 1997.

[22] S. Bowong and F. M. Moukam Kakmeni, "Chaos control and duration time of a class of uncertain chaotic systems," Physics Letters A, vol. 316, no. 3-4, pp. 206-217, 2003.

[23] X. Wang, H. Wu, and J. Cao, "Global leader-following consensus in finite time for fractional-order multi-agent systems with discontinuous inherent dynamics subject to nonlinear growth," Nonlinear Analysis: Hybrid Systems, vol. 37, Article ID 100888, 2020.

[24] Y. Jia, H. Wu, and J. Cao, "Non-fragile robust finite-time synchronization for fractional-order discontinuous complex networks with multi-weights and uncertain couplings under asynchronous switching," Applied Mathematics and Computation, vol. 370, Article ID 124929, 2020.

[25] M. Liu, H. Wu, and W. Zhao, "Event-triggered stochastic synchronization in finite time for delayed semi-Markovian jump neural networks with discontinuous activations," Computational and Applied Mathematics, vol. 39, p. 118, 2020.

[26] X. Yang and J. Cao, "Finite-time stochastic synchronization of complex networks," Applied Mathematical Modelling, vol. 34, no. 11, pp. 3631-3641, 2010.

[27] Y. Zhang, H. Wu, and J. Cao, "Group consensus in finite time for fractional multiagent systems with discontinuous inherent dynamics subject to hölder growth," IEEE Transactions on Cybernetics, vol. 1, 2020.

[28] A. Polyakov, "Nonlinear feedback design for fixed-time stabilization of linear control systems," IEEE Transactions on Automatic Control, vol. 57, no. 8, pp. 2106-2110, 2012.

[29] W. Zhao and H. Wu, "Fixed-time synchronization of semiMarkovian jumping neural networks with time-varying delays," Advances in Difference Equations, vol. 2018, p. 213, 2018.

[30] C. Hu, J. Yu, Z. Chen, H. Jiang, and T. Huang, "Fixed-time stability of dynamical systems and fixed-time synchronization of coupled discontinuous neural networks," Neural Networks, vol. 89, pp. 74-83, 2017.

[31] Z. Zuo, "Nonsingular fixed-time consensus tracking for second-order multi-agent networks," Automatica, vol. 54, pp. 305-309, 2015.

[32] M. Zheng, L. Li, H. Peng et al., "Fixed-time synchronization of memristive fuzzy BAM cellular neural networks with timevarying delays based on feedback controllers," IEEE Access, vol. 6, p. 1, 2018.

[33] Y. Huang, S. Qiu, S. Ren, and Z. Zheng, "Fixed-time synchronization of coupled cohen-grossberg neural networks with and without parameter uncertainties," Neurocomputing, vol. 315, pp. 157-168, 2018.

[34] C. Chen, L. Li, H. Peng, J. Kurths, and Y. Yang, "Fixed-time synchronization of hybrid coupled networks with timevarying delays," Chaos, Solitons \& Fractals, vol. 108, pp. 49$56,2018$.

[35] X. Zhu, X. Yang, F. E. Alsaadi, and T. Hayat, "Fixed-Time Synchronization of coupled discontinuous neural networks 
with nonidentical perturbations," Neural Processing Letters, vol. 48, no. 2, pp. 1161-1174, 2018.

[36] Z. Wang and H. Wu, "Global synchronization in fixed time for semi-Markovian switching complex dynamical networks with hybrid couplings and time-varying delays," Nonlinear Dynamics, vol. 95, no. 3, pp. 2031-2062, 2019.

[37] H. Lv and W. He, "Fixed-time synchronization for coupled delayed neural networks with discontinuous or continuous activations," Neurocomputing, vol. 314, pp. 143-153, 2018.

[38] A. Filippov, Differential Equations with Discontinuous RightHand Sides, Kluwer, Dordrecht, The Netherlands, 1988.

[39] S. Ding and Z. Wang, "Event-triggered synchronization of discrete-time neural networks: a switching approach," Neural Networks, vol. 125, 2020.

[40] S. Ding, Z. Wang, and N. Rong, "Intermittent control for quasisynchronization of delayed discrete-time neural networks," IEEE Transactions on Cybernetics, vol. 99, pp. 1-12, 2020.

[41] M. Forti and P. Nistri, "Global convergence of neural networks with discontinuous neuron activations," IEEE Transactions on Circuits and Systems I: Fundamental Theory and Applications, vol. 50, no. 11, pp. 1421-1435, 2003.

[42] M. Liu and H. Wu, "Stochastic finite-time synchronization for discontinuous semi-Markovian switching neural networks with time delays and noise disturbance," Neurocomputing, vol. 310, pp. 246-264, 2018.

[43] J. Aubin and A. Cellina, Differential Inclusions, Spring-Verlag, Berlin, Germany, 1984.

[44] M. Forti, P. Nistri, and D. Papini, “Global exponential stability and global convergence in finite time of delayed neural networks with infinite gain," IEEE Transactions on Neural Networks, vol. 16, no. 6, pp. 1449-1463, 2005.

[45] J. Liu, H. Wu, and J. Cao, "Event-triggered synchronization in fixed time for semi-Markov switching dynamical complex networks with multiple weights and discontinuous nonlinearity," Communications in Nonlinear Science and Numerical Simulation, vol. 90, Article ID 105400, 2020.

[46] J. Liu, H. Wu, and J. Cao, "Event-triggered synchronization in fixed time for complex dynamical networks with discontinuous nodes and disturbances," Mathematical Modelling in Computational and Life Sciences, vol. 3, no. 38, pp. 2503-2515, 2020.

[47] C. Murguia, J. Ruths, and H. Nijmeijer, "Robust network synchronization of time-delayed coupled systems," IFACPapersOnLine, vol. 49, no. 14, pp. 74-79, 2016.

[48] X. Peng and $\mathrm{H}$. Wu, "Non-fragile robust finite-time stabilization and $\mathrm{H} \infty$ performance analysis for fractional-order delayed neural networks with discontinuous activations under the asynchronous switching," Neural Computing and Applications, vol. 32, no. 8, pp. 4045-4071, 2020. 